\title{
Sensibilité des enseignant-es aux facteurs scolaires de risque de décrochage
}

\section{Anne-Françoise de Chambrier, Valérie Angelucci, Léonie Liechti et Oliver Prosperi}

Le décrochage scolaire, phénomène complexe et processuel, est le résultat de facteurs tant internes qu'externes au système scolaire. La présente étude vise à analyser la conscience qu'ont les enseignant-es de l'importance des variables scolaires dans le risque de décrochage, ainsi que l'influence de certaines de leurs caractéristiques sur ces représentations. Les résultats montrent que les enseignant-es sont moyennement sensibles aux facteurs scolaires de risque de décrochage, cela assez indépendamment de leurs caractéristiques. Ils attribuent davantage d'importance aux facteurs propres à l'élève et à son milieu familial, mais à ce niveau plusieurs différences apparaissent en fonction des caractéristiques des enseignant-es.

\section{Introduction}

Le décrochage scolaire préoccupe indéniablement les instances politiques et scolaires tant le poids du diplôme est conséquent dans le monde du travail exigeant et compétitif d'aujourd'hui. Objet de nombreux débats conceptuels (Bernard, 2014; Esterle-Hedibel, 2006), la notion de décrochage scolaire est mobilisée dans le cadre de cet article afin de favoriser une compréhension globale des difficultés de l'élève en lien avec son environnement sans pour autant évacuer la question des apprentissages (Bernard, 2013). Alors que la quantification du décrochage scolaire renvoie aux jeunes sortant prématurément du système éducatif sans qualification ${ }^{1}$, nous adoptons ici une approche fondamentalement interactionniste. Cette dernière nous conduit de fait à adhérer au consensus qui se dessine dans le champ scientifique, considérant le décrochage comme un processus et non un résultat (Boudesseul, 2013). Le décrochage est alors compris comme une expérience scolaire processuelle, mais non forcément linéaire, marquée par des difficultés d'apprentissage (un décrochage cognitif) précédant de fréquents problèmes de comportement et d'attitude pouvant conduire à de l'absentéisme et à une disparition précoce du système scolaire (Bernard, 2014; Boudesseul, 2013; Bryk \& Thum, 1989; Terrail, 2002). Le décrochage est 
ainsi un problème résolument complexe aux origines multifactorielles et résulte surtout de la combinaison et de l'accumulation de différents facteurs de risque qui déclenchent ou entretiennent le processus du décrochage (Blaya, 2010).

\section{Multiplicité et poids des facteurs de risque du décrochage scolaire}

Les spécialistes dans le domaine s'accordent tant sur la précocité de l'émergence des difficultés que sur la multiplicité des facteurs de risque, ceux-ci pouvant relever de l'individu, de sa famille, de l'école et plus largement de la société. La classification des déterminants du décrochage scolaire proposée par Gilles, Tièche Christinat et Delévaux (2012) distingue à ce sujet deux catégories principales (déterminants externes et déterminants internes au système scolaire), ellesmêmes divisées en deux sous-catégories selon le schéma suivant (Figure 1).
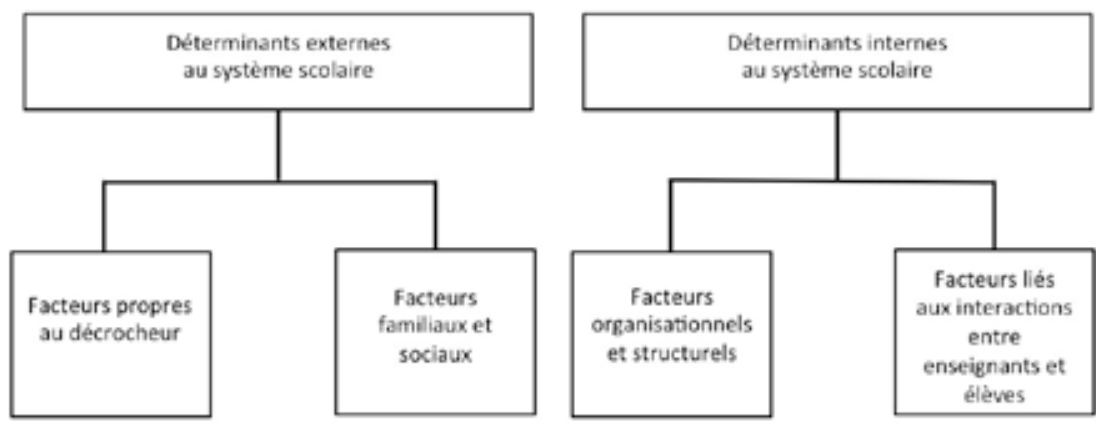

Figure 1: Classification des facteurs de risque du décrochage scolaire adaptée de Gilles et al., 2012.

Parmi les facteurs propres aux jeunes en décrochage figurent par exemple la dépression (Gagné, Marcotte \& Fortin, 2011), les difficultés comportementales (Fortin, Marcotte, Potvin, Royer \& Joly 2006), ou le trouble de l'attention (Gregg, 2009). Pour les facteurs familiaux et sociaux, la pauvreté (Kaufman, Alt \& Chapman, 2004), les familles désunies ou recomposées, des parents faiblement scolarisés (Astone \& McLanahan, 1991; Potvin et al., 1999) ou une faible implication de la part des parents dans les activités scolaires (Fortin, Marcotte, Diallo, Potvin \& Royer, 2013) représentent des paramètres fortement associés au décrochage. D'autres auteurs ont mis en évidence le poids de variables scolaires dans le décrochage, pointant une inadaptation réciproque entre le système scolaire et la population à laquelle il s'adresse. Ainsi, dans les facteurs organisationnels et structurels propres au système scolaire, on sait notamment que les pratiques d'exclusion temporaire et celle du redoublement (Crahay, 2004) sont sources importantes de décrochage scolaire, tout comme la transition entre le primaire et le secondaire (Bautier, 2003; Bonnéry, 2003) ou l'existence de filières de relégation (Lafontaine \& Crahay, 2004). Concernant les facteurs liés aux interactions entre 
élèves et enseignant-es, les études montrent que le nombre élevé d'intervenant-es dans une classe, l'absentéisme, l'ennui, le climat scolaire voire certaines pratiques pédagogiques (étiquetage, gestion des temps morts,...) sont fortement associés au risque de décrochage scolaire (Blaya 2010; Esterle-Hedibel, 2006; Gilles et al., 2012). Ce genre de constat amène Cahuc et ses collègues à dépeindre l'école comme une véritable «machine à trier» (Cahuc, Carcillo, Galland \& Zylberberg, 2011) échouant dans sa mission d'offrir «à tous les jeunes un accès à l'école et des chances égales d'émancipation sociale» (Gilles et al. 2012, p.4). Quant à l'importance relative de ces différents facteurs, et si le processus est bien le fruit de l'accumulation de plusieurs d'entre eux, Blaya (2010) relève que les variables scolaires, telles que le climat scolaire, le manque de clarté des règles, la perception de la capacité d'innovation chez les enseignant-es et le soutien des enseignant-es, sont les plus significatives. De nombreuses données montrent pourtant que les enseignant-es n'attribuent que peu d'importance aux facteurs scolaires, considérant davantage l'influence des facteurs relevant de l'individu et de sa famille. Ce constat questionne tant l'importance considérable des représentations que les enseignant-es se font au sujet de leurs élèves est connue à ce jour.

\section{Représentations au sujet de la difficulté scolaire et caractéristiques du corps enseignant}

Différents travaux se sont effectivement attachés à mettre en évidence l'importance des croyances, perceptions et attentes des enseignant-es envers leurs élèves, celles-ci contribuant à orienter les pensées et comportements des enseignant-es à l'égard de ces derniers. Ces croyances et perceptions peuvent même entraîner une modification de la perception que les élèves ont d'eux-mêmes et les conduire à se conformer à la croyance de l'enseignant-e en modifiant leur engagement dans l'activité (Bressoux \& Pansu, 2003). L'impact des représentations que les enseignant-es peuvent avoir de leurs élèves est ainsi connu sous le terme d' «effet des attentes» ou, dans le domaine éducatif plus particulièrement, d' «effet Pygmalion", et ce depuis les travaux princeps de Rosenthal et Jacobson (1968). Cet effet désigne l'influence positive de croyances sur le potentiel d'évolution scolaire d'un-e élève et sur ses aptitudes. Cette recherche a fait l'objet de critiques, notamment en raison de biais méthodologiques (Bressoux \& Pansu, 2003; Jensen, 1969; Thorndike, 1968) et de la faible puissance des résultats (Trouilloud \& Sarrazin, 2003). Elle fait néanmoins partie de ce que Merton (1948) a désigné sous le terme de prophéties autoréalisatrices, et les différentes méta-analyses conduites à ce sujet (e.g., Cooper \& Hazelrigg, 1988; Rosenthal \& Rubin, 1978) en ont toutes souligné les effets, même modestes.

Ainsi, les questions relatives aux représentations des enseignant-es envers leurs élèves sont cruciales et ont été étudiées au sujet de l'échec scolaire, du redoublement, de l'intelligence des élèves, et, dans de moindres mesures, au sujet des facteurs de risque du décrochage scolaire. De nombreux travaux montrent que les enseignant-es ont essentiellement tendance à attribuer les 
difficultés scolaires des élèves à des causes exogènes à l'école. Les explications des enseignant-es mettent en avant des déficits propres aux élèves ou des difficultés liées à l'environnement socio-familial, mais rarement le fonctionnement institutionnel ou les pratiques des enseignant-es (Beckers, 1994; Bless, Bonvin \& Schüpbach, 2005; Bourgeois, 1983; Dréano \& Gaillard, 2002; Gossot \& Dubreuil, 2003; Talbot, 2006). Relevons toutefois que quelques études sur les représentations des enseignant-es vis-à-vis de l'échec scolaire font apparaître que certaines variables scolaires sont considérées comme autant voire plus influentes que certains facteurs externes au système scolaire. Par exemple, l'étude de Bouchamma (2002) montre que les enseignant-es se sentent significativement moins responsables de l'échec que l'étudiant-e, mais plus responsables comparativement à l'école et à la société. Aïssani (2008) met pour sa part en évidence que dans leurs explications de l'échec scolaire, les enseignant-es expriment de façon assez nette une mise en cause de l'institution scolaire, notamment par le manque d'équipement des écoles, le contenu des programmes, les rythmes scolaires, les méthodes d'enseignement, les attitudes négatives ou le manque de soutien de la part de l'enseignant-e. Les quelques données concernant plus spécifiquement les représentations des enseignant-es sur les causes du décrochage scolaire ont plutôt mis en évidence une tendance à sous-estimer l'importance des facteurs d'ordre scolaire. L'étude de Parent, Duquette et Carrier (1993) montre par exemple que les enseignant-es attribuent plus d'influence aux facteurs famille, élève et société (grande influence) qu'aux facteurs structure, pédagogie, enseignant-es et direction (influence moyenne). Les résultats concernant l'importance attribuée aux facteurs d'ordre scolaire dans les représentations des enseignant-es sur les causes des difficultés scolaires ne se montrent donc pas très concordants et les données relatives au risque de décrochage scolaire sont de toute évidence insuffisantes.

Par ailleurs, si en aval, les représentations influencent la pratique pédagogique, en amont, ces croyances semblent reliées à certaines caractéristiques des enseignant-es (Pouliot \& Potvin, 2000). En effet, l'influence de plusieurs de ces dernières a été mise en évidence dans des travaux sur les représentations des enseignant-es au sujet par exemple du rendement scolaire ou du redoublement (Bouchamma, 2002, Potvin \& Rousseau, 1993, Pouliot \& Potvin, 2000). Plusieurs caractéristiques des enseignant-es semblent influencer significativement leurs représentations, mais les résultats en la matière divergent. Par exemple, en ce qui concerne le genre, tantôt les femmes attribuent plus d'importance que les hommes au rôle de l'enseignant-e (Guskey, 1981; Scherer \& Kimmel, 1993), tantôt ce sont les hommes qui allouent à l'enseignant-e plus de responsabilité dans l'échec scolaire alors que les femmes incriminent plus l'élève (Bouchamma, 2002; Potvin \& Papillon, 1992) et tantôt les représentations se montrent peu influencées par le genre de l'enseignant-e (Gilly, 2003). Les représentations des enseignant-es semblent également dépendre de l'âge et/ou de l'expérience professionnelle de celles-ci ou ceux-ci. Les résultats tendent plutôt à montrer que plus 
les enseignant-es sont âgé-es, plus ils accordent de l'importance aux facteurs externes au système scolaire (Berthelot, 1991; Bourgeois, 1983). L'influence de l'expérience professionnelle s'est quant à elle souvent révélée significative, mais peu concordante quant aux attributions que les enseignant-es font sur le rendement scolaire (voir Bouchamma, 2002).

Le niveau de qualification semble également représenter une variable pertinente au niveau de l'influence qu'elle peut avoir sur les représentations des enseignant-es. Concernant le rendement scolaire, Bouchamma (2002) souligne que les enseignant-es plus qualifié-es auraient tendance à se considérer plus responsables de l'échec que ne le ferait un-e enseignant-e possédant une moins grande qualification. Au sujet du redoublement, Pouliot et Potvin (2000) relèvent que les enseignant-es détenant un grade universitaire sont significativement moins favorables au redoublement que les enseignant-es détenant un brevet. Cette caractéristique des enseignant-es nous intéresse tout particulièrement étant donné les changements qui ont eu lieu dans la formation des enseignant-es en Suisse et notamment dans le canton de Vaud il y a maintenant une dizaine d'années ${ }^{2}$. Perrenoud soulignait d'ailleurs en 1993 qu'une des raisons majeure d'accélérer la professionnalisation des enseignant-es - au sens entre autres d'une activité savante et d'une formation longue - résidait dans la nécessité de lutter contre l'échec scolaire et les inégalités devant l'école. Nous voulons donc vérifier si les enseignant-es au bénéfice d'une formation tertiaire se montrent plus conscients de l'importance des facteurs scolaires de risque du décrochage.

Finalement, toujours concernant les représentations des enseignant-es quant aux facteurs scolaires de risque de décrochage, l'influence du degré d'enseignement (primaire ou secondaire) des enseignant-es nous intéresse également plus particulièrement. En effet, comme présenté plus haut, si le processus de décrochage scolaire commence souvent tôt dans la scolarité, le degré secondaire est un moment particulièrement délicat en matière de risque de décrochage scolaire, que ce soit fonction de la difficulté que représente la transition primaire-secondaire (Anderson, Jacobs, Schramm \& Plittgerber, 2000), de la vulnérabilité propre à l'adolescence (Favresse \& Piette, 2004) ou de la sélection qui s'opère dans le fonctionnement de ce degré scolaire (Lafontaine \& Crahay, 2004). Par ailleurs, en Suisse et notamment dans le canton de Vaud, le parcours de formation des enseignant-es est spécifique à chaque degré d'enseignement. Si les enseignant-es primaires ont généralement suivi soit l'ancienne école normale, soit une haute école pédagogique, les enseignant-es du secondaire sont en général titulaires d'une première formation universitaire dans leurs disciplines d'enseignement ainsi que d'une formation professionnelle de niveau tertiaire leur permettant d'enseigner aux élèves de 12 à 15 ans. Nous souhaitons vérifier si les enseignant-es du degré secondaire, de par leur cursus de formation différent et leur confrontation quotidienne à un cycle de la scolarité particulièrement délicat en termes de risque de décrochage scolaire, se distinguent par une plus grande sensibilité aux facteurs d'ordre scolaire dans le risque de décrochage. 
Ainsi, puisque les recherches montrent que plusieurs variables scolaires influencent notablement le risque de décrochage et puisqu'il est avéré que les représentations des enseignant-es retentissent sur les comportements des élèves, il est primordial de mieux connaitre la conscience qu'ont les enseignant-es de l'importance de ces facteurs scolaires dans le risque de décrochage. Par ailleurs, étant donné qu'il semble peu pertinent de considérer les enseignant-es comme un groupe homogène et qu'au contraire leurs représentations sont liées à plusieurs de leurs caractéristiques, nous étudions ici combien le genre, les années d'expérience professionnelle, le type de formation (tertiaire ou non) et le degré d'enseignement (primaire ou secondaire) ont une incidence sur la sensibilité des enseignant-es quant aux facteurs scolaires de risque de décrochage.

\section{Méthode}

Dans le but d'explorer les représentations du corps enseignant quant aux différents facteurs influençant le décrochage scolaire, un questionnaire en ligne a été élaboré et diffusé auprès de 2724 enseignant-es des degrés primaire et secondaire du canton de Vaud (Suisse) par le biais des directions des établissements scolaires et via la plateforme électronique officielle de communication de support à l'enseignement «educanet2». Sa complétion s'est déroulée de septembre à décembre 2012, incluant des relances à deux reprises auprès des directions des établissements scolaires de la part de l'équipe de recherche.

\section{Instrument}

La présente étude s'inscrit dans une recherche plus large sur le décrochage scolaire $^{3}$, et seules les données issues de son volet quantitatif sont ici utilisées. Elles proviennent d'un questionnaire élaboré à partir de l'analyse de dix entretiens semi-directifs et exploratoires menés auprès d'enseignant-es romand-es, d'une revue de la littérature portant sur les facteurs de risque avérés du décrochage scolaire (Bautier, 2003; Blaya, 2010; Broccolichi, 2000; Fortin \& Picard, 1999; Fortin, Marcotte, Potvin, Royer \& Joly, 2006; Glasman, 2000; Janosz, 2000; Janosz \& Leblanc, 2005; Parent et al., 1993; Rumberger \& Ah Lim, 2008) et de la classification des déterminants du décrochage scolaire de Gilles et al. (2012).

La première section du questionnaire s'intéresse aux caractéristiques sociodémographiques et professionnelles des répondant-es, et en particulier à leur genre, type de formation (non-tertiaire ou tertiaire), nombre d'années d'expérience en enseignement, et degré d'enseignement actuel (primaire ou secondaire). La seconde section du questionnaire porte sur les représentations des enseignant-es vis-à-vis des facteurs de risque avérés du décrochage scolaire. Trente-deux items de type "les difficultés de comportement (agressivité, indiscipline) influencent le décrochage scolaire» ont été soumis à l'examen des partici- 
pant-es et ont été encodés sur une échelle de type Likert à six échelons (de "pas du tout d'accord» à «tout à fait d'accord»).

Le questionnaire a été testé avec un échantillon de trente répondant-es (enseignant-es du canton de Vaud et étudiant-es de la Haute école pédagogique du canton de Vaud). Cette évaluation qualitative (Laveault \& Grégoire, 2005) a permis de clarifier la formulation de certains éléments (consignes, questions, items, échelle) et d'optimiser l'interface.

\section{Échantillon}

À la fin de la période de complétion du questionnaire en ligne, 700 questionnaires ont pu être collectés, assurant ainsi un taux de retour de $26 \%$. En raison de données manquantes dans la deuxième section du questionnaire, 71 questionnaires ont été exclus. L'échantillon se compose donc de 629 enseignant-es dont presque $20 \%$ sont des hommes (contre $27 \%$ pour l'ensemble de la population enseignante du canton de Vaud). L'échantillon se compose à $66 \%$ d'enseignant-es du degré primaire. La moyenne d'âge de l'échantillon se situe à 42 ans $(\mathrm{ET}=10.07)$ et le nombre d'années d'expérience à $18.27(\mathrm{ET}=10.3)$. Enfin, 27\% des enseignant-es ont suivi une formation tertiaire.

\section{Présentation des résultats}

Afin de répondre à nos questions de recherche, une analyse en composantes principales (Acp) a tout d'abord été menée sur les items du questionnaire. Ensuite, les résultats concernant les représentations générales des enseignant-es vis-à-vis des facteurs de risque du décrochage scolaire sont rapportés, suite auxquels des t-tests pour échantillons indépendants et des analyses de variance univariées (Anova) ont été conduits afin d'analyser les éventuelles différences de représentations des enseignant-es vis-à-vis des différents facteurs liées au décrochage scolaire selon leurs caractéristiques. Les répondant-es présentant des données manquantes concernant les variables sociodémographiques concernées ont été exclu-es des analyses.

\section{Résultats de L'analyse en composantes principales} L'Acp (rotation Varimax avec normalisation de Kaiser) a permis de retenir 22 items sur 32 qui ont été regroupés en six composantes et nommés de la manière suivante: 1) aspects comportementaux et émotionnels propres à l'élève; 2) engagement éducatif parental; 3) capital socio-éducatif; 4) contraintes pédagogiques et structurelles; 5) gestion de l'établissement scolaire; 6) vécu scolaire difficile (Tableau 1). La variance totale expliquée est de 53\%. Les alphas de Cronbach sont satisfaisants à l'exception de la composante relative à la gestion de l'établissement scolaire.

Tel que synthétisé dans le Tableau 1, les six composantes obtenues par l'Acp ont été regroupées en trois dimensions relativement à la classification de Gilles 
et al. (2012). Ainsi, les composantes «aspects comportementaux et émotionnels propres à l'élève», «engagement éducatif parental» et "capital socio-éducatif» font précisément partie de ce que Gilles et al. incluent dans les déterminants externes au système scolaire. Les composantes "contraintes pédagogiques et structurelles» et "gestion de l'établissement scolaire» relèvent clairement des déterminants internes au système scolaire. Quant à la composante "vécu scolaire difficile», elle fait apparaitre une dimension supplémentaire par rapport à la classification de Gilles et al. que nous avons nommée hybride. En effet, cette composante issue de l'Acp, constituée des items «difficultés d'apprentissage», "performances scolaires faibles», expérience scolaire précédente négative», et "attitudes négatives de l'enseignant-e envers les élèves», paraît appartenir tant à la dimension externe au système scolaire (en l'occurrence aux facteurs propres au décrocheur) qu'à sa dimension interne (plus spécifiquement aux facteurs liés aux interactions entre enseignant-es et élèves).

\section{Représentations générales du corps enseignant vis-à-vis des facteurs de risque du décrochage scolaire}

Le Tableau 1 indique les alphas de Cronbach, moyennes et écarts-types des scores obtenus par item, composante et dimension. La dimension hybride obtient le score moyen le plus élevé $(\mathrm{M}=4.58 ; \mathrm{ET}=0.60)$, suivie de près par la dimension externe au système scolaire $(\mathrm{M}=4.46 ; \mathrm{ET}=0.57)$ et, finalement, par la dimension interne au système scolaire $(\mathrm{M}=3.58 ; \mathrm{ET}=0.60)$.

Au niveau des composantes, on constate que ce sont, par ordre d'importance, les aspects comportementaux et émotionnels propres à l'élève, le vécu scolaire difficile, l'engagement parental éducatif ainsi que la gestion de l'établissement scolaire qui présentent les scores moyens les plus élevés (score moyen supérieur à la valeur médiane de l'échelle située à 3.5). Les composantes "capital socioculturel» et «contraintes pédagogiques et structurelles» présentent pour leur part une moyenne inférieure ou égale à 3.5. 
Tableau 1: Alphas de Cronbach, moyennes et écarts-types des scores obtenus pour chaque item retenu par l'Acp, pour les composantes qui les regroupent et pour les dimensions auxquelles celles-ci ont été rattachées en référence au modèle de Gilles et al. (2012).

\begin{tabular}{|c|c|c|c|}
\hline \multicolumn{2}{|l|}{$\mathrm{N}=629$} & M & ET \\
\hline \multicolumn{2}{|c|}{ Dimension interne au système scolaire } & 3.58 & 0.60 \\
\hline \multicolumn{2}{|c|}{$\begin{array}{l}\text { Composante: gestion de l'établissement scolaire } \\
\alpha \text { de Cronbach: } 0.52\end{array}$} & 3.93 & 0.99 \\
\hline \multirow[t]{2}{*}{ Items: } & & 3.68 & 1.21 \\
\hline & $\begin{array}{l}\text { L’insuffisance des ressources humaines disponibles (ensei- } \\
\text { gnant.e.s, psychologues, logopédistes, conseillers et conseillères } \\
\text { en orientation, travailleuses et travailleurs sociaux...) }\end{array}$ & 4.19 & 1.18 \\
\hline \multicolumn{2}{|c|}{$\begin{array}{l}\text { Composante: contraintes pédagogiques et structurelles } \\
\alpha \text { de Cronbach: } \mathbf{0 . 6 6}\end{array}$} & 3.46 & 0.64 \\
\hline \multirow[t]{5}{*}{ Items: } & Une gestion du comportement à l'école par la sanction & 3.40 & 1.08 \\
\hline & Des devoirs à domicile trop exigeants & 3.52 & 1.01 \\
\hline & Des exigences de programme trop élevées & 3.52 & 1.10 \\
\hline & L'exigence d'autonomie accrue au secondaire & 3.48 & 0.98 \\
\hline & Les mesures d'exclusion temporaires (jours de suspension, etc.) & 3.62 & 1.02 \\
\hline \multicolumn{2}{|c|}{ Dimension externe au système scolaire } & 4.46 & 0.57 \\
\hline \multicolumn{2}{|c|}{$\begin{array}{l}\text { Composante: aspects comportementaux et émotionnels propres à l'élève } \\
\alpha \text { de Cronbach: } 0.78\end{array}$} & 4.88 & 0.65 \\
\hline \multirow[t]{5}{*}{ Items: } & Les conduites délinquantes en dehors de la classe & 5.07 & 0.87 \\
\hline & Les difficultés de comportement de l'élève (agressivité, indiscipline) & 4.87 & 0.89 \\
\hline & L'absentéisme & 5.03 & 0.88 \\
\hline & Le niveau d'engagement scolaire faible de la part de l'élève & 4.71 & 0.91 \\
\hline & Le caractère dépressif de l'élève & 4.73 & 0.89 \\
\hline \multicolumn{2}{|c|}{$\begin{array}{l}\text { Composante: engagement parental éducatif } \\
\alpha \text { de Cronbach: } 0.71\end{array}$} & 4.42 & 0.71 \\
\hline \multirow[t]{4}{*}{ Items: } & La faible implication scolaire de la part des parents & 4.64 & 0.92 \\
\hline & L'environnement socio-culturel de l'élève & 4.19 & 1.05 \\
\hline & De faibles attentes en termes de réussite scolaire de la part des parents & 4.15 & 1.00 \\
\hline & Un encadrement éducatif à domicile déficient & 4.70 & 0.92 \\
\hline \multicolumn{2}{|c|}{$\begin{array}{l}\text { Composante: capital socio-culturel } \\
\alpha \text { de Cronbach: } 0.71\end{array}$} & 3.50 & 1.14 \\
\hline \multirow[t]{2}{*}{ Items: } & Un bon niveau de scolarisation des parents & 3.35 & 1.21 \\
\hline & Une bonne intégration sociale de la famille & 3.66 & 1.38 \\
\hline \multicolumn{2}{|c|}{ Dimension hybride } & 4.58 & 0.60 \\
\hline \multicolumn{2}{|c|}{$\begin{array}{l}\text { Composante: vécu scolaire difficile } \\
\alpha \text { de Cronbach: } 0.65\end{array}$} & 4.58 & 0.60 \\
\hline \multirow[t]{4}{*}{ Items: } & Les difficultés d'apprentissage & 4.67 & 0.84 \\
\hline & Les performances scolaires faibles & 4.43 & 0.84 \\
\hline & Une expérience scolaire précédente négative & 4.41 & 0.83 \\
\hline & Des attitudes négatives de l'enseignant-e envers les élèves & 4.81 & 0.94 \\
\hline
\end{tabular}


Représentations du corps enseignant vis-à-vis des facteurs de risque du décrochage scolaire selon leurs caractéristiques

Les résultats significatifs et tendanciels concernant les différences de représentations des enseignant-es en fonction de leurs caractéristiques socio-démographiques et professionnelles sont présentés pour les composantes de chacune des dimensions.

\section{Composantes liées à la dimension interne au système scolaire}

Au niveau des composantes relatives à la dimension interne au système scolaire, on observe que relativement peu de groupes d'enseignant-es se distinguent en fonction de leurs caractéristiques. Les résultats permettent de relever une tendance des femmes à considérer davantage que les hommes les contraintes pédagogiques et structurelles comme influençant le risque de décrochage scolaire $\left(\mathrm{M}_{\text {femmes }}=3.49, \mathrm{ET}=.62\right.$ vs. $\left.\mathrm{M}_{\text {hommes }}=3.37, \mathrm{ET}=.73 ; t(627)=1.846, p=.065\right)$. Quant aux caractéristiques professionnelles des enseignant-es, les enseignant-es occupant un poste au degré secondaire sont ceux qui attribuent le moins d'importance aux contraintes pédagogiques et structurelles comme influençant le risque de décrochage scolaire $\left(M_{\text {secondaire }}=3.36, E T=0.67\right.$ vs. $M_{\text {primaire }}=3.52$, $\mathrm{ET}=0.62 ; t(627)=3.067, p=.002, d=0.25)$. Aucune différence significative n'est révélée en ce qui concerne le type de formation (tertiaire ou non) ni le nombre d'années d'expérience professionnelle.

\section{Composantes liées à la dimension externe au système scolaire}

Au niveau des composantes relatives à la dimension externe au système scolaire, il ressort que les hommes considèrent davantage que les femmes l'influence de l'engagement parental éducatif $\left(M_{\text {hommes }}=4.61, E T=.68, M_{\text {femmes }}=4.37\right.$, $\mathrm{ET}=.71, t(627)=3.321, p=.001, d=.33)$ et du capital socio-culturel de la famille $\left(\mathrm{M}_{\text {hommes }}=3.78, \mathrm{ET}=1.17, \mathrm{M}_{\mathrm{femmes}}=3.43, \mathrm{ET}=1.13, t(627)=3.109, p=.002\right.$, $d=.31)$ sur le risque du décrochage scolaire.

Au niveau des caractéristiques professionnelles des enseignant-es, les résultats indiquent que les représentations des facteurs de risque du décrochage scolaire se distinguent selon le nombre d'années d'expérience en enseignement. En effet, les répondant-es ayant le plus d'années d'expérience attribuent davantage d'importance que leurs collègues moins expérimenté-es aux aspects comportementaux et émotionnels propres à l'élève $\left(\mathrm{M}_{21}\right.$ ans et plus $=4.95, \mathrm{ET}=.61, \mathrm{M}_{20}$ ans et moins $=4.82$ $\mathrm{ET}=.67, t(623)=2.479, p=.013, d=.20)$ et tendanciellement plus d'importance à l'engagement parental éducatif $\left(\mathrm{M}_{21}\right.$ ans et plus $=4.48, \mathrm{ET}=.71, \mathrm{M}_{20}$ ans et moins $=4.37, \mathrm{ET}=.71, t(623)=1.890, p=.059, \mathrm{~d}=.16)$. Les enseignant-es avec 20 ans et moins d'expérience considèrent pour leur part davantage le capital socioculturel de la famille que leurs collègues $\left(\mathrm{M}_{20}\right.$ ans et moins $=3.59, \mathrm{ET}=1.06, \mathrm{M}_{21}$ ans et plus $=3.36, \mathrm{ET}=1.23, t(513.722)=2.414, p=.019, d=.20)$. Par ailleurs, les enseignant-es formé-es au niveau tertiaire considèrent comme moins influents 
les aspects comportementaux et émotionnels de l'élève que les enseignant-es formé-es au niveau non-tertiaire $\left(\mathrm{M}_{\text {tertiaire }}=4.73, \mathrm{ET}=.70, \mathrm{M}_{\text {non-tertiaire }}=4.93\right.$, $\mathrm{ET}=.62, t(627)=3-560, p=.000, d=.32)$, mais ils attribuent plus d'influence au capital socio-culturel des parents que leurs collègues formé-es au niveau non-tertiaire $\left(\mathrm{M}_{\text {tertiaire }}=3.66, \mathrm{ET}=1.08, \mathrm{M}_{\text {non-tertiaire }}=3.44, \mathrm{ET}=1.16, t(627)=2.100\right.$, $p=0.36, d=.19$ ). Finalement, les enseignant-es qui occupent un poste au degré secondaire accordent plus d'importance que les enseignant-es qui occupent un poste au degré primaire à l'influence de l'engagement parental éducatif $\left(\mathrm{M}_{\text {secon- }}\right.$ daire $\left.=4.56, \mathrm{ET}=.71, \mathrm{M}_{\text {primaire }}=4.35, \mathrm{ET}=.70, t(627)=3.517, p=.000, d=.30\right)$ et au capital socio-culturel des parents (respectivement et $\mathrm{M}_{\text {secondaire }}=3.68, \mathrm{ET}=1.15$, $\left.\mathrm{M}_{\text {primaire }}=3.41, \mathrm{ET}=1.13, t(627)=2.848, p=.005, d=.24\right)$.

\section{Composantes liées à la dimension hybride}

Concernant les composantes relatives à la dimension hybride, les tests effectués indiquent que les enseignant-es avec le moins d'expérience en enseignement accordent tendanciellement plus d'importance au vécu scolaire difficile que leurs collègues $\left(\mathrm{M}_{20}\right.$ et moins $=4.61, \mathrm{ET}=.59$ vs. $\mathrm{M}_{21 \text { et plus }}=4.53, \mathrm{ET}=.62, t(623)=1.654$, $p=.099)$.

\section{Discussion}

Comme nous l'avons vu en introduction, le processus de décrochage est autant si ce n'est plus (Blaya, 2010; Blaya \& Fortin, 2011) - lié à des facteurs internes au système scolaire qu'externes à celui-ci. Etant donné l'importance avérée des représentations des enseignant-es et la mesure dans laquelle celles-ci sont influencées par certaines caractéristiques des enseignant-es, il importe de bien comprendre l'importance qu'attribuent les différents groupes d'enseignant-es aux facteurs scolaires de risque de décrochage par rapport aux facteurs externes.

\section{Représentations des enseignant-es quant à l'importance des facteurs scolaires de risque de décrochage}

Notre étude montre de façon générale que les enseignant-es ont tendance à sous-estimer l'influence des variables d'ordre scolaire dans le risque de décrochage. En effet, la dimension interne au système scolaire (gestion de l'établissement scolaire et contraintes pédagogiques et structurelles) obtient le score moyen le plus bas, à peine au-dessus de la valeur médiane de l'échelle du questionnaire. Ceci est d'autant plus vrai pour les contraintes pédagogiques et structurelles du système scolaire, qui regroupent des items tels qu'une gestion du comportement à l'école par la sanction, une exigence d'autonomie accrue au secondaire, les mesures d'exclusion temporaire ou des devoirs à domicile trop exigeants. Plus particulièrement, il est intéressant de relever que si l'absentéisme (item associé suite à l'Acp aux aspects comportementaux et émotionnels propres 
à l'élève) est l'un des deux items de notre questionnaire considéré comme influençant le plus le risque de décrochage, les mesures d'exclusion temporaires (associées aux contraintes pédagogiques et structurelles du système scolaire) sont à l'inverse considérées comme un des facteurs les moins influents, et ce malgré les conséquences négatives avérées de l'exclusion temporaire (Moignard, 2014). Parmi les facteurs scolaires, l'insuffisance des ressources humaines disponibles est le seul item à être considéré comme plus influent. Relevons toutefois que les enseignant-es sont globalement tout-à-fait sensibles à l'influence des difficultés d'apprentissage, aux performances scolaires faibles et aux attitudes négatives de l'enseignant-e envers les élèves, items ressortis de l'Acp comme étant associés à la dimension que nous avons nommée hybride (à la fois interne et externe au système scolaire).

Au niveau des caractéristiques des enseignant-es, nous pouvons regretter que les enseignant-es bénéficiant d'une formation tertiaire ne se distinguent pas par une plus grande sensibilité à l'importance de ces facteurs scolaires. En effet, si la formation tertiaire des enseignant-es peut être considérée comme un niveau de professionnalisation et de qualification plus élevée, celle-ci ne se traduit pas par une plus grande sensibilité aux facteurs scolaires de risque de décrochage. Nous pouvons également relever que contrairement à nos attentes, les enseignant-es du degré secondaire ne se distinguent pas non plus par une plus grande conscience des risques liés à certaines caractéristiques de leurs degrés scolaires, cycle qui comme nous l'avons vu en introduction est un moment particulièrement sensible en termes de risque de décrochage scolaire. Ainsi, bien qu'étant régulièrement au front de la vulnérabilité de leurs élèves adolescent-es et de ce fonctionnement institutionnel, les enseignant-es du degré secondaire se distinguent à l'inverse par une moins grande importance attribuée aux contraintes pédagogiques et structurelles de l'école en termes de facteurs de risque du décrochage. Seules les femmes se montrent tendanciellement plus sensibles à l'influence de cette dernière composante.

\section{Représentations des enseignant-es quant à l'importance des facteurs externes de risque de décrochage}

Les facteurs externes au système scolaire sont globalement considérés par nos répondant-es comme influençant plus le risque de décrochage scolaire, plus particulièrement en ce qui concerne les aspects comportementaux et émotionnels propres à l'élève et l'engagement parental éducatif, et moins concernant le capital socio-culturel. Notons cependant que c'est au sein de cette dimension externe au système scolaire que les avis sont les plus partagés en fonction des caractéristiques des enseignant-es.

Concernant le nombre d'années d'expérience professionnelle, les enseignant-es plus expérimenté-es incriminent davantage les aspects comportementaux et émotionnels propres à l'élève et tendanciellement plus l'engagement parental éducatif. Les enseignant-es moins expérimenté-es considèrent pour 
leur part le capital socio-culturel comme plus influent (et tendanciellement plus le vécu scolaire difficile de la dimension hybride). Il se pourrait donc que la tendance à incriminer les facteurs propres à l'élève comme source de décrochage s'accroisse avec l'expérience professionnelle.

Les enseignant-es bénéficiant d'une formation tertiaire attribuent pour leur part moins d'importance aux aspects comportementaux et émotionnels propres à l'élève et plus d'importance au capital socio-culturel. Ainsi, si comme susmentionné il peut être déploré que les enseignant-es au bénéfice d'une formation tertiaire ne sont pas plus conscient-es de l'influence des facteurs scolaires, nous pouvons toutefois nous réjouir du fait que les enseignant-es ayant été formé-es en haute école ne commettent pas du tout ce que Ross (1977) a appelé l'erreur fondamentale d'attribution. Celle-ci consiste à sous-estimer les causes situationnelles au profit des causes dispositionnelles, et est aussi parfois appelée biais d'internalité (attribuer systématiquement à l'individu la responsabilité de sa conduite). Ainsi, les enseignant-es formé-es au niveau tertiaire considèrent à juste titre que l'élève n'est en tout cas pas seul responsable de son décrochage scolaire, mais que celui-ci est notamment fonction des interactions entre l'élève et son milieu socio-culturel.

Dans le même sens, les hommes et les enseignant-es du secondaire se distinguent également par une plus grande importance attribuée à l'engagement parental éducatif et au capital socio-culturel. Relevons donc que si - comme évoquée plus haut - les enseignant-es du secondaire se montrent moins sensibles aux contraintes pédagogiques et structurelles internes au système scolaire, ils sont cependant très sensibles au poids du milieu comme facteur de risque de décrochage.

\section{Une sensibilité peu élevée aux poids du capital socio-culturel et du fonction- nement de l'institution scolaire}

En résumé et en référence à la classification des déterminants du décrochage scolaire de Gilles et al. (2012), nous pouvons relever que les enseignant-es considèrent en général le décrochage scolaire comme relevant plus des déterminants externes qu'internes au système scolaire. Cependant, une analyse plus précise des résultats à la lumière des quatre sous-catégories de la classification de Gilles et al. (facteurs propres au décrocheur et facteurs familiaux et sociaux pour les déterminants externes au système scolaire; facteurs organisationnels et structurels et facteurs liés aux interactions entre enseignant-es et élèves pour les déterminants internes au système scolaire) met en évidence des nuances en ce qui concerne l'influence de ces deux types de déterminants. Au sein des déterminants externes au système scolaire, nos résultats montrent que les enseignant-es incriminent davantage les facteurs propres au décrocheur et les facteurs familiaux que les facteurs sociaux. En effet, malgré les différences susmentionnées relatives aux caractéristiques des enseignant-es, l'influence de la composante capital socioculturel, constituée des items «bon niveau de scolarisation des parents» et «bonne 
intégration sociale de la famille», a récolté auprès de notre échantillon un score d'approbation peu élevé. Ce résultat va donc plutôt dans le sens de ceux de Crahay et Ory (2006) qui démontrent que les futur-es enseignant-es "persistent à nier le poids de l'influence de l'origine socioculturelle des élèves dans la fabrication des échecs scolaires» (cité par Crahay, Wanlin, Issaieva \& Laduron, 2010, p. 101).

Quant aux deux sous-catégories des déterminants internes au système scolaire de la classification de Gilles et al. (2012), notre étude montre que ce sont surtout les facteurs organisationnels et structurels de l'école dont les enseignant-es sous-estiment l'influence. En effet, les facteurs liés aux interactions entre enseignant-es et élèves de la classification de Gilles et al. peuvent être considérés comme des déterminants internes au système scolaire auxquels les enseignant-es sont plus sensibles. C'est du moins ce que suggère l'item «attitude négative de l'enseignant-e envers l'élève» qui apparaît parmi ceux ayant reçu le plus haut degré d'accord des enseignant-es quant à leur influence sur le décrochage scolaire. C'est donc plus précisément de l'impact du fonctionnement général de l'institution scolaire en tant que générateur effectif de décrochage scolaire que les enseignant-es de notre échantillon semblent peu conscient-es, et ce d'autant plus pour les enseignant-es du secondaire.

Ainsi, si nos données suggèrent que plusieurs facteurs scolaires sont considérés comme moins importants que les facteurs individuels et familiaux dans le risque de décrochage, leur interprétation en termes de biais d'auto-complaisance (Miller \& Ross, 1975) n'est pas suffisante pour expliquer ces résultats. Ce biais consiste à attribuer sa propre réussite à des causes internes (auto-attribution) et son échec à des causes externes (hétéro-attribution) afin de maintenir une image positive de soi. Ce phénomène peut indirectement et en partie expliquer que les enseignant-es tendent à imputer l'échec de leurs élèves à des causes extra-scolaires ou propres à l'élève. Toutefois, parmi les items que l'Acp a permis de retenir, certains impliquant directement l'enseignant-e ont été considérés comme peu influents (mesures d'exclusion temporaire, gestion du comportement par la sanction) alors que d'autres ont été jugés tout à fait influents (attitudes négatives de l'enseignant-e envers les élèves). Ainsi, plutôt que d'éviter de remettre leurs compétences en cause, la tendance des enseignant-es à sous-estimer certains facteurs scolaires comme source de décrochage témoigne probablement plus d'une difficulté à considérer le fonctionnement général du système scolaire (exigences des programmes, autonomie accrue au secondaire, soutien de la direction) - dont ils ne sont pas responsables - comme facteur contribuant au décrochage. Ces résultats convergent donc avec ceux de Crahay et Ory (2006) qui concluaient notamment que les futur-es enseignant-es, en fin de formation comme en début, rechignent à mettre en cause le fonctionnement général de l'école (cité par Crahay, Wanlin, Issaieva \& Laduron, 2010).

Finalement, certaines limites de l'étude nécessitent d'être relevées, permettant également de suggérer des perspectives pour des recherches futures. Tout 
d'abord, la structure et les dimensions inférées par l'Acp pourraient ne pas être stables étant donné qu'une procédure de validation par bootstrapping n'a pas été menée dans cette étude. De plus, il n'est pas possible d'affirmer que la taille de l'échantillon est suffisante, bien que selon Field (2009) un échantillon supérieur à 250 sujets permette une bonne stabilité de la méthode de détermination des dimensions dans ce type d'analyse. De ce fait, il serait pertinent d'élargir l'étude en couvrant l'ensemble de la population romande des enseignant-es, permettant également de consolider et d'améliorer l'instrument de mesure élaboré pour les fins de cette étude.

\section{Conclusion}

Cette recherche - qui vise à étudier l'importance qu'attribuent les enseignant-es aux facteurs scolaires de risque de décrochage, et a fortiori le rôle que leur reconnaissent les enseignant-es au bénéfice d'une formation tertiaire et les enseignant-es du secondaire - amène des éléments nouveaux dans ce champ de connaissances. Nos résultats montrent que les enseignant-es considèrent de façon assez homogène les facteurs scolaires comme influençant moins le risque de décrochage que les aspects comportementaux et émotionnels propres à l'élève et que l'engagement parental éducatif. Ceci est d'autant plus vrai pour les contraintes pédagogiques et structurelles de l'école et de la part des enseignant-es du secondaire.

Le faible poids attribué par les enseignant-es aux facteurs organisationnels et structurels internes au système scolaire semble montrer qu'un effort doit être fait pour informer et sensibiliser le personnel scolaire sur l'importance de ces variables dans le processus de décrochage. Dans cette optique, une plus grande sensibilisation dans la formation initiale ou continue des enseignant-es aux variables scolaires qui génèrent du décrochage est très vraisemblablement de mise. La faible importance attribuée à ces facteurs scolaires soulève les enjeux relatifs à la formation des enseignant-es quant à son impact sur les représentations des enseignant-es et in fine sur leurs pratiques pédagogiques.

\section{Notes}

1 En Suisse, sont considérés comme décrocheurs les "Jeunes âgés de 18 à 24 ans sans formation post-obligatoire et qui ne sont plus scolarisés. Jeunes quittant prématurément l'école» (Office Fédérale des Statistiques, 2017).

2 Le premier type (non-tertiaire) correspond à la formation telle qu'elle était dispensée dans le canton de Vaud jusqu'au début des années 2000 (système de l'école normale). Depuis la création de la Haute école pédagogique du canton (2001), les diplômes correspondent aux grades universitaires appliqués suivant le système de Bologne (Bachelor, Master). Ainsi, les personnes formées depuis sont considérées comme titulaires d'un diplôme de formation tertiaire.

3 Cette recherche a été possible grâce à la subvention du Fonds National Suisse de la Recherche Scientifique n 13 DPD3-136975 


\section{Bibliographie}

Aïssani, Y. (2008). Explication de l'échec scolaire et représentation sociale. Journal for Communication Studies, 1, (1-2), 66-77.

Anderson, L. W., Jacobs, J., Schramm, S. \& Splittgerber, F. (2000). School transitions: beginning of the end or a new beginning? International Journal of Educational Research, 33, 325-339.

Astone, N. M. \& McLanahan, S. S. (1991). Family structure, parental practices, and high school completion. American Sociological Review, 56, (3), 309-320.

Bautier, É. (2003). Décrochage scolaire: Genèse et logique des parcours. VEI Enjeux, 132, $30-45$.

Beckers, J. (1994). Modifier les représentations de l'échec à l'École normale. Recherche en éducation: théorie et pratique, 17, 3-13.

Bernard, P.-Y. (2013). Le décrochage scolaire. Paris: Presses universitaires de France.

Bernard, P.-Y. (2014). Le décrochage scolaire en France: usage du terme et transformation du problème scolaire. Carrefours de l'Education, 1, (37), 29-45.

Berthelot, M. (1991). Enseigner: qu'en disent les profs? (Rapport d'une recherche menée auprès du personnel enseignant du primaire, du secondaire et du collégial). Québec: Conseil supérieur de l'éducation.

Blaya, C. (2010). Décrochages scolaires. L'école en difficulté. Bruxelles: De Boeck.

Blaya, C. \& Fortin, L. (2011). Les élèves français et québécois à risque de décrochage scolaire: comparaison entre les facteurs de risque personnels, familiaux et scolaires. L'orientation scolaire et professionnelle, 40, (1), 1-26.

Bless, G., Bonvin, P. \& Schüpbach, M. (2005). Le redoublement scolaire: ses déterminants, son efficacité, ses conséquences. Bern: Haupt.

Bonnéry, S. (2003). Le décrochage scolaire de l'intérieur: interaction de processus sociaux cognitifs, subjectifs et langagiers. Les Sciences de l'Éducation. Pour l'Ėre Nouvelle, 26, (1), 39-58.

Bouchamma, Y. (2002). Relation entre les explications de l'échec scolaire et quelques caractéristiques d'enseignants du collégial. Revue des Sciences de l'Éducation, 28, (3), 649-674.

Boudesseul, G. (2013). Introduction. In G. Boudesseul (Éd.), Du décrochage à la réussite scolaire: expériences française et internationales, 7-16. Paris: Editions L'Harmattan.

Bourgeois, J.-P. (1983). Comment les instituteurs perçoivent l'échec scolaire. Revue Française de Pédagogie, 62, 27-39.

Bressoux, P. \& Pansu, P. (2003). Quand les enseignants jugent leurs élèves. Paris: Presses Universitaires de France.

Broccolichi, S. (2000). Désagrégation des liens pédagogiques et situations de rupture. VEI Enjeux, 122, 36-47.

Bryk, A. S. \& Thum, Y. M. (1989). The effects of high school organization on dropping out: An exploratory investigation. American Educational Research Journal, 26, (3), 353-383.

Cahuc, P., Carcillo, S., Galland, O. \& Zylberberg, A. (2011). La machine à trier: comment la France divise sa jeunesse. Paris: Eyrolles.

Cooper, H. M. \& Hazelrigg, P. (1988). Personality moderators of interpersonal expectancy effects: An integrative research review. Journal of Personality and Social Psychology, 6, 937-949.

Crahay, M. (2004). Peut-on conclure à propos des effets du redoublement? Revue Française de Pédagogie, 148, 11-24.

Crahay, M. \& Ory, P. (2006, mars). Les représentations des normaliens en matière de redoublement évoluent-elles au cours de leur formation à l'école normale? Communication présentée au 4 congrès des chercheurs en éducation, Bruxelles.

Crahay, M., Wanlin, P., Issaieva, E. \& Laduron, I. (2010). Fonction, structuration et évolution des croyances (et connaissances) des enseignants. Revue Française de Pédagogie, 172, 85-129. 
Dréano, C. \& Gaillard, B. (2002). Étude des représentations du psychologue chez les enseignants dans une perspective d'aide aux enfants en difficulté à l'école. Psychologie et Education, 51, 79-54.

Esterle-Hedibel, M. (2006). Absentéisme, déscolarisation, décrochage scolaire, les apports des recherches récentes. Déviance et Société, 30, 41-65.

Favresse, D. \& Piette, D. (2004). Les jeunes en marge du système scolaire: inscription dans une socialisation de l'exclusion. L'Observatoire, 43, 87-91

Field, A. (2009). Discovering statistics using SPSS. London: SAGE.

Fortin, L. \& Picard, Y. (1999). Les élèves à risque de décrochage scolaire: facteurs discriminants entre décrocheurs et persévérants. Revue des Sciences de l'Éducation, 25, (2), 359-374.

Fortin, L., Marcotte, D., Diallo, T., Potvin, P. \& Royer, É. (2013). A multidimensional model of school dropout from an 8-year longitudinal study in a general high school population. European Journal of Psychology of Education, 28, (2), 563-583.

Fortin, L., Marcotte, D., Potvin, P., Royer, E. \& Joly, J. (2006). Typology of students at risk of dropping out of school: description by personal, family and school factors. European Journal of Psychology of Education, 21, 363-383.

Gagné, M.-E., Marcotte, D. \& Fortin, L. (2011). L'impact de la dépression et de l'expérience scolaire sur le décrochage scolaire des adolescents. Revue Canadienne de l'Éducation, 34, (2), 77-92.

Gilles, J.-L., Tièche Christinat, C. \& Delévaux, O. (2012). Origines, fondements et perspectives offertes par les alliances éducatives dans la lutte contre le décrochage scolaire. In J.-L. Gilles, P. Potvin \& C. Tièche Christinat (Éd.), Les alliances éducatives pour lutter contre le décrochage scolaire (pp. 3-18). Bern: Peter Lang.

Gilly, M. (2003). Les représentations sociales dans le champ éducatif. In D. Jodelet (Éd.), Les représentations sociales (pp. 383-486). Paris: Presses Universitaires de France.

Glasman, D. (2000). Le décrochage scolaire: une question sociale et institutionnelle. VEI Enjeux, 122, 10-25.

Gossot, B. \& Dubreuil, P. (2003). Les élèves en difficulté à l'entrée du collège (Rapport de la commission "Élèves en difficulté» $n^{\circ}$ 03-033). Paris: Inspection générale de l'Éducation nationale, MEN.

Gregg, N. (2009). Adolescents and adults with learning disabilities and ADHD: Assessment and accomodation. New York: The Guilford Press.

Guskey, T. R. (1981). Measurement of the responsibility teachers assume for academic succes and failures in the classroom. Journal of Teacher Education, 31, (3), 44-51.

Janosz, M. (2000). L'abandon scolaire chez les adolescents: perspective nord-américaine. VEI Enjeux, 122, 105-127.

Janosz, M. \& Leblanc, M. (2005). L'abandon scolaire à l'adolescence: des déterminants communs aux trajectoires multiples. In G. Brandibas \& R. Fourasté (Éd.), Les accidentés de l'école (pp. 67-97). Paris: L'Harmattan.

Jensen, A. R. (1969). How much can we boost I.Q. and scholastic achievement? Harvard Educational Review, 29, 1-123.

Kaufman, P., Alt, M. N. \& Chapman, C. (2004). Dropout rates in the United States: 2001. Washington, US: Department of Education.

Lafontaine, D. \& Crahay, M. (2004). Échec et décrochage scolaires en Communauté française de Belgique. Revue Internationale d'Éducation de Sèvres, 35, 55-66.

Laveault, D. \& Grégoire, J. (2005). Introduction aux théories des tests en psychologie et en sciences de l'éducation (2 éd.). Bruxelles: De Boeck.

Merton, R. (1948). The self-fulfilling prophecy. Antioch Review, 8, 193-210.

Miller, D. T. \& Ross, M. (1975). Self-serving biases in the attribution of causality. Fact or fiction? Psychological Bulletin, 82, 213-225.

Moignard, B. (2014). Le collège fantôme: une mesure de l'exclusion temporaire des collégiens. Diversité, 175, 1-8. 
Office Fédérale des Statistiques (2017). Jeunes quittant prématurément l'école. Retrieved from https://www.bfs.admin.ch/bfs/fr/home/statistiques/education-science/indicateurs-formation/indicateurs/décrochage-scolaire.htlm.

Parent, G., Duquette, R. \& Carrier, J. (1993). Opinions des enseignants sur les causes du décrochage scolaire. Revue des Sciences de l'Education, 19, (3), 537-553.

Perrenoud, P. (1993). Formation initiale des maîtres et professionnalisation du métier. Revue des sciences de l'éducation, 19, (1), 59-76.

Potvin, P., Deslandes, R., Beaulieu, P., Marcotte, D., Fortin, L., Royer, É. \& Leclerc, L. (1999). Le risque d'abandon scolaire en lien avec le style parental et la participation parentale au suivi scolaire. Revue Canadienne de l'Éducation, 24, (4), 441-453.

Potvin, P. \& Papillon, S. (1992). Teachers' sense of responsibility toward students achievement and their attitude. Canadian Journal of Special Education, 8, (1) 33-42.

Potvin, P. \& Rousseau (1993). Attitudes des enseignants envers les élèves en difficulté scolaire. Revue canadienne de l'éducation, 18, (2), 132-149.

Pouliot, L. \& Potvin, P. (2000). Croyances d'enseignants de la maternelle et du primaire à l'égard du redoublement. Revue canadienne de l'éducation, 25, (4), 247-261.

Rosenthal, R. \& Jacobson, I. (1968). Pygmalion in the classroom: teacher expectation and pupils intellectual development. New York: Holt, Rinehart and Winston.

Rosenthal, R. \& Rubin, D. B. (1978). Interpersonal expectancy effects: The first 345 studies. The Behavioral and Brain Sciences, 3, 377-386.

Ross, L. (1977). The intuitive psychologist and his shortcomings: Distortions in the attribution process. In L. Berkowitz (Éd.), Advances in Experimental Social Psychology (pp. 173-220). New York: Academic Press.

Rumberger, R. \& Ah Lim, S. (2008). Why students drop out of school: a review of 25 years of research (California Dropout research project). Santa Barbara: University of California.

Scherer, M. E. \& Kimmel, E. (1993). Modifying teachers' attributions: an education consultation approach. Communication présentée à la 39e Rencontre de l'Association de psychologie, Atlanta.

Talbot, L. (2006). Les représentations des difficultés d'apprentissage chez les professeurs des écoles. Empan, 63, 49-56.

Terrail, J. P. (2002). Prologue. Décrochage scolaire et décrochage cognitif. Décrochage scolaire: genèse et logique des parcours (Rapport de recherche pour la Direction de la Programmation et du Développement). Paris: Ministère de l'Education nationale, de l'Enseignement supérieur et de la Recherche.

Thorndike, R. L. (1968). Review of Pygmalion in the classroom. American Educational Research Journal, 5, 708-711.

Trouilloud, D. \& Sarrazin, P. (2003). Les connaissances actuelles sur l'effet Pygmalion: processus, poids et modulateurs. Revue française de pédagogie, 145, 89-119.

Mots-clés: Décrochage scolaire, facteurs de risque, variables scolaires, représentations des enseignant-es, caractéristiques des enseignant-es 


\section{Das Bewusstein von Lehrpersonen zu schulischen Risikofaktoren bei Schulabbruch}

\section{Zusammenfassung}

Der Schulabbruch ist ein komplexes, schrittweise eintretendes Phänomen und das Resultat sowohl interner als auch externer Faktoren des Schulsystems. Die vorliegende Studie will das Bewusstsein von Lehrpersonen betreffend der schulischen Risikofaktoren, sowie den Einfluss von Merkmalen der Lehrpersonen auf diese Vorstellung, analysieren. Die Resultate zeigen dass Lehrpersonen sich der schulischen Faktoren mittelmässig bewusst sind und dies ziemlich unabhängig von Lehrermerkmalen. Sie erachten die Eigenschaften der Schülerinnen und Schüler sowie deren Familienumgebung als wichtiger, obwohl sich auch Differenzen in Bezug auf bestimmte Lehrermerkmale finden.

Schlagworte: Schulabbruch, Risikofaktoren, schulische Faktoren, Lehrpersonen, Vorstellungen, Lehrermerkmale

\section{Consapevolezza degli insegnanti dei fattori scolastici del rischio di abbandono scolastico}

\section{Riassunto}

L'abbandono scolastico, un fenomeno complesso e processuale, è il risultato di fattori sia interni sia esterni al sistema scolastico. Questa ricerca ha come obbiettivo da un lato l'analisi della consapevolezza da parte degli insegnanti dell'importanza delle variabili scolastiche in relazione al rischio di abbandono scolastico, ma anche lo studio dell'influenza di alcune loro caratteristiche su queste rappresentazioni. I risultati mostrano che gli insegnanti sono relativamente sensibili ai fattori scolastici di rischio d'abbandono e ciò indipendentemente dalle loro caratteristiche. Attribuiscono infatti più importanza ai fattori propri degli allievi e al contesto familiare, anche se a questo livello emergono parecchie differenze in funzione delle caratteristiche dell'insegnante.

Parole chiave: Abbandono scolastico, fattori di rischio, variabili scolastiche, rappresentazioni degli insegnanti, caratteristiche degli insegnanti 


\section{Teachers' Sensivity to School Dropout Risk Factors}

\section{Summary}

School dropout is a complex and process phenomenon; it results from both internal and external school factors. This study aims to analyse teachers' awareness of the importance those risk factors play in school dropout, and how some of their characteristics might influence their representations. The results generally show that, independently of their characteristics, teachers are moderately aware of school related factors in school dropping out. They attach greater importance to student related factors and to their family environment, although some differences depending on teachers' characteristics emerge.

Keywords: School dropout, risk factors, related to school risk factors, teacher representations, teacher characteristics 